\section{An Empirical Transmitted EPID Dosimetry Method using a Back- Projection Algorithm}

\author{
Hashemi S. M. ${ }^{1 \oplus}$, Bahreyni M. H. ${ }^{1}$, Mohammadi M. ${ }^{2,3}$, Nas- \\ seri S. ${ }^{1}$, Bayani S. ${ }^{4}$, Gholamhosseinian H. ${ }^{1}$, Salek R. ${ }^{5}$, Shahe- \\ di F. ${ }^{6}$, Momennezhad M. ${ }^{1,7 * \odot}$
}

\begin{abstract}
Background: The present study aimed to introduce a rapid transmission dosimetry through an electronic portal-imaging device (EPID) to achieve two-dimensional (2D) dose distribution for homogenous environments.

Material and Methods: In this Phantom study, first, the EPID calibration curve and correction coefficients for field size were obtained from EPID and ionization chamber. Second, the EPID off-axis pixel response was measured, and the greyscale image of the EPID was converted into portal dose image using the calibration curve. Next, the scattering contribution was calculated to obtain the primary dose. Then, by means of a verified back-projection algorithm and the Scatter-to-Primary dose ratio, a 2D dose distribution at the mid-plane was obtained.
\end{abstract}

Results: The results obtained from comparing the transmitted EPID dosimetry to the calculated dose, using commercial treatment planning system with gamma function while there is $3 \%$ dose difference and $3 \mathrm{~mm}$ distance to agreement criteria, were in a good agreement. In addition, the pass rates of $\gamma<1$ was $94.89 \%$ for the homogeneous volumes.

Conclusion: Based on the results, the method proposed can be used in EPID dosimetry.

Citation: Hashemi S. M, Bahreyni M. H, Mohammadi M, Nasseri S, Bayani S, Gholamhosseinian H, Salek R, Shahedi F, Momennezhad M. An Empirical Transmitted EPID Dosimetry Method using a Back-Projection Algorithm. J Biomed Phys Eng. 2019;9(5):551-558. https://doi.org/10.31661/jbpe.v0i0.1082.

\section{Keywords}

Radiotherapy Planning, Computer-Assisted; Dose-Response Relationship; Radiotherapy; Algorithms

\section{Introduction}

$\mathrm{P}$ atient-specific treatment verification is an unavoidable trend with the current complexity of modern radiotherapy plans and dose prescriptions [1]. Different devices have been used for the verification. Not only Electronic Portal Imaging Devices (EPIDs) are developed for patient position verification, but also they can be used for other tasks such as dosimetry and quality assurance [2,3]. Currently, the most common type of EPID available is the amorphous-silicon EPID (a-Si EPID) [1]. The advantages of a-Si EPID are as follows: positional accuracy, stability, high spatial resolution, real-time image acquisition, and signal digitization capabilities $[4,5]$. On the other hand, the over
${ }^{1}$ PhD, Medical Physics Research Center, Mashhad University of medical Science, Mashhad, Iran

${ }^{2} \mathrm{PhD}$, Department of Medical Physics, Royal

Adelaide Hospital, Ade-

laide, SA 5000, Australia

${ }^{3} \mathrm{PhD}$, School of Physical

Sciences, University of

Adelaide, Adelaide, SA

5005, Australia

${ }^{4} \mathrm{MSc}$, Medical Phys-

ics Research Center,

Mashhad University of

medical Science, Mash-

had, Iran

${ }^{5} \mathrm{MD}$, Cancer Research

Center, Mashhad University of medical Science,

Mashhad, Iran

${ }^{6} \mathrm{MSc}$, Department of Radiology, School of Para-

medical Sciences, Torbat

Heydariyeh University of

Medical Sciences, Torbat

Heydariyeh, Iran

${ }^{7}$ PhD, Nuclear Medi-

cine Research Center,

Mashhad University of

medical Science, Mash-

had, Iran

*Corresponding author: M. Momennezhad Medical Physics Research Center, Mashhad University of medical Science, Mashhad, Iran E-mail: Momennezhadm@mums.ac.ir

Received: 12 December 2018 Accepted: 25 January 2019 
sensitivity to low-energy photons is the main disadvantage of this device [6].

Several researchers have showed the short and long-term stabilities [6-10]. The response of a-Si EPIDs is reportedly independent of dose rate and approximately linear with the dose delivered [11]. Regarding the mentioned advantages above, a-Si EPIDs are suitable devices for both pre-treatment and treatment (in vivo) verification approaches [12].

The EPID dosimetry can be performed in both transit and non-transit models [1]. The transit dosimetry is the measurement of the dose behind the patient/phantom at the detector plane, and the non-transit dosimetry, is the determination of dose without an attenuating medium between the linear accelerator's target and EPID [1]. Given the incapability of the non-transited model in detecting all errors during the treatment, the transit model is preferred over the non-transit one [13]. In both models, EPID dosimetry can be performed by either forward or backward approaches [14]. In the forward approach, the measured portal image can be converted into the transmitted dose using different methods. The Portal Dose Images (PDI) can then be compared with dose distribution in EPID position calculated by a treatment planning system (TPS) or another method like Monte Carlo simulation [15-17]. In contrast, in the backward approach (or back-projection), the measured electronic portal images (EPIs) are used to reconstruct the patient dose in the treatment position at any plane [18].

There are different studies investigating the transmitted EPID dosimetry for dose determination at the patient level using the back-projection methods $[8,18-20]$. Transit dosimetry based on the EPID has been also compared with those calculated with commercial TPS. The results indicated that there was a good agreement on gamma index analysis for the homogenous and anthropomorphic phantoms [21].

This study aims to perform the transmission dosimetry in clinical workflow, in a way that the isocentric dose plane at the patient's position is estimated using back projecting of the exit energy fluency recorded by the EPID. The results obtained were only for mid-plane dose; however, with regard to the applied calculation method, the 2D dose map could be calculated for any source-to-image distance (SID) using the same procedure. Therefore, the results can be generalized to any distance from the accelerator.

\section{Material and Methods}

In this Phantom study, the measurements were made on a Precise linear accelerator (Elekta Oncology Systems, Crawley, UK) with a multileaf collimator, consisting of 40 leaf pairs with $1 \mathrm{~cm}$ width and the source to axis distance of $100 \mathrm{~cm}$. The detector panel was the PerkinElmer Amorphous Silicon (aSi) with IviewGT supporting software. More details can be found in the machine's manual [22].

The integrated pixel value for each field was obtained using Equation 1:

Integrated PixelValue $=\frac{65535-\text { RawPixelValue }}{P S F}(1)$

Where, PSF is the pixel-scaling factor, related to the number of frames for each image $[23,24]$.

\section{A. Calibration of EPID}

To determine the relationship between EPID signal and ion chamber, EPIs were obtained from a slab phantom, $20 \mathrm{~cm}$, by setting a $10 \times 10 \mathrm{~cm}^{2}$ radiation field size and delivering the varying number of MU (5-150 MUs). The homogeneous slab phantom was placed on the treatment couch at a source-to-detector distance (SDD) of $160 \mathrm{~cm}$.

The procedure was repeated with the same scenario for a calibrated 0.6 cc Farmer ionization chamber that was inserted in the EPID position at the maximum distance of $1.5 \mathrm{~cm}$ from the slabs. 
These processes were repeated to determine the field size corrections for EPID and ion chamber when the slab phantoms, $20 \mathrm{~cm}$, was irritated with $50 \mathrm{MU}$ in $4 \times 4,5 \times 5,7 \times 7,10 \times 10$ and $15 \times 15 \mathrm{~cm}^{2}$ field sizes. All fields were normalized to a standard field size $10 \times 10 \mathrm{~cm}^{2}$ and the curves were then fitted.

\section{B. Beam hardening and scatter cor- rection for water medium}

The attenuation function for primary dose in water was expressed as follows $[25,20]$ :

$$
A\left(T_{\text {Mid }}\right)=\exp \left(-\mu T_{\text {Mid }}\left(1-\eta T_{\text {Mid }}\right)\right)
$$

Where $\eta$ is the beam-hardening coefficient. The above equation can be written as:

$$
A\left(T_{\text {Mid }}\right)=\exp \left(-\mu_{H d} T_{\text {Mid }}\right)
$$

Where

$$
\mu_{H d}=\mu\left(1-\eta T_{\text {Mid }}\right)
$$

Where $\mu_{\mathrm{Hd}}$ represents the linear attenuation coefficient applying beam hardening, and $\mathrm{T}_{\text {Mid }}$ denotes the thickness matrix obtained for the mid-plane. The $\mu_{\mathrm{Hd}}$ matrix was obtained from both mid-plane and the EPID position. To determine the ratio of scattered radiation in dose distribution, the scatter-to-primary dose ratio (SPR) reported by WANG et al. was employed using the following equation [26].

$$
S P R=\frac{a_{0} s\left(T_{\text {Mid }}+d_{0}\right)}{w_{0} s+\left(T_{\text {Mid }}+d_{0}\right)}
$$

Where, $\mathrm{a}_{0}, \mathrm{w}_{0}$, and $\mathrm{d}_{0}$ are fitting parameters on the linear attenuation coefficient $(\mu)$, and $s$ is the field size.

\section{Off-axis correction for EPID}

First, the portal image was obtained from slab phantom, $20 \mathrm{~cm}$, placing on the couch with delivering a $25 \times 25 \mathrm{~cm}^{2}$ irradiation field to correct the off-axis response of EPID. The EPID image was normalized to the center. Considering, the beam divergence, the matrix of beam attenuation was calculated for $20 \mathrm{~cm}$ thickness of slabs using the attenuation function (Equation 3) by MATLAB software R2016b (Mathworks Inc., Natick, Massachusetts, USA), then the result was normalized to the center. Second, the normalized EPID matrix determined at the first step was multiplied pixel-wise by the normalized attenuation function to obtain the off-axis response.

\section{Back-Projection method and ver- ification}

The method obtains the dose delivered to the mid plane by means of the back-projection algorithm involving, a) the calculation of the primary dose by means of EPID and b) its back projection to the mid plane and applying the SPR [18]. In the first step, the equations obtained for the EPID field size correction in section A were utilized to calculate the primary dose for the intended field size. At the second stage, the following function was run:

$$
\text { PrDose }_{\text {Mid }}=\text { PrDose }_{E P I D}\left(\frac{d_{E P I D}}{d_{\text {Mid }}}\right)^{2} A\left(T_{\text {Mid }}\right)
$$

Where $\operatorname{PrDose}_{E P I D}$ and PrDose Mid $_{\text {are the }}$ matrixes of the primary dose at EPID position and mid-plane, respectively. Furthermore, $d_{E-}$ ${ }_{P I D}$ and $d_{\text {Mid }}$ are the matrix distances from the accelerator target to EPID and mid-plane, respectively.

\section{E. verification of the Method}

For the verification of the method in a homogeneous volume, a slab phantom, $20 \mathrm{~cm}$, was located on the treatment couch and irradiated with $60 \mathrm{MU}$ at a field size of $8 \times 8 \mathrm{~cm}^{2}$. Gafchromic EBT3 film was used to evaluate the penumbra region at the same beam configuration.

\section{Results}

\section{A. EPID Calibration}

Equation 7 reveals the linear relationship between the dose at the EPID position and the mean pixel value acquired from EPI central point of the slab phantoms for the field size of 
a $10 \times 10 \mathrm{~cm}^{2}$.

Dose $(c G y)=3.26 \times 10^{(-5)} \times$ Pixel Value $+0.02787(7)$

Based on the quadratic functions, beam scat- tering is dependent on the radiation field size. Equations 8 and 9 present the quadratic functions obtained from the field size effects when the slab phantoms, $20 \mathrm{~cm}$,were placed on the couch for the EPID and Ion chamber.

F.S Scatter Factor for EPID $=-5.65 \times 10^{(-5)} \times s^{2}+2.12 \times 10^{(-2)} \times s+7.92 \times 10^{(-1)}$

F.S Scatter Factor for Ion Chamber $=-5.276 \times 10^{(-5)} \times s^{2}+1.63 \times 10^{(-2)} \times s+8.404 \times 10^{(-1)}$

Where, $s$ is the desired field size.

\section{B. Beam hardening and scattering Correction}

Figure 1a displays the linear attenuation coefficient map $\left(\mu_{\mathrm{Hd}}\right)$ used to compensate beam hardening at the mid-plane for the slab phantom using Equation 4. In this equation, the data

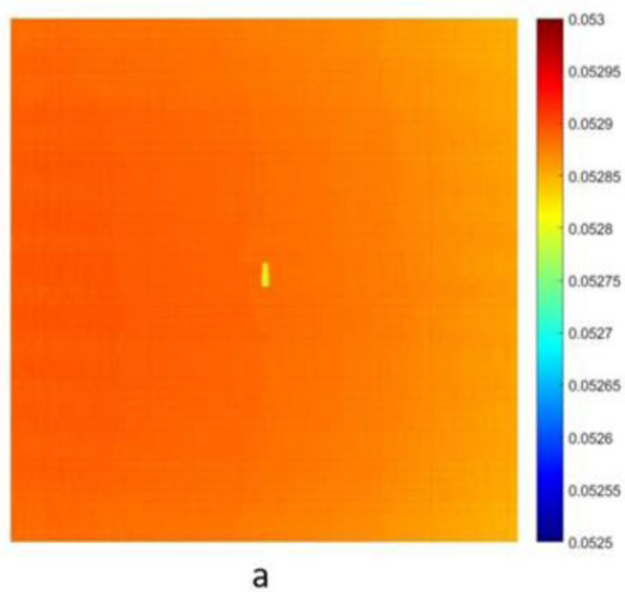

presented by WANG et al., for various Linac and beam energies calculated using the Monte Carlo simulation method [26] were used for the $\mu$ and $\eta$ parameters. The $\mu_{\mathrm{Hd}}$ map for midplane is shown in Figure 1a.

Figure $1 \mathrm{~b}$ demonstrates the obtained SPR based on Equation 5. This matrix was developed for the determination of the scatter radia-

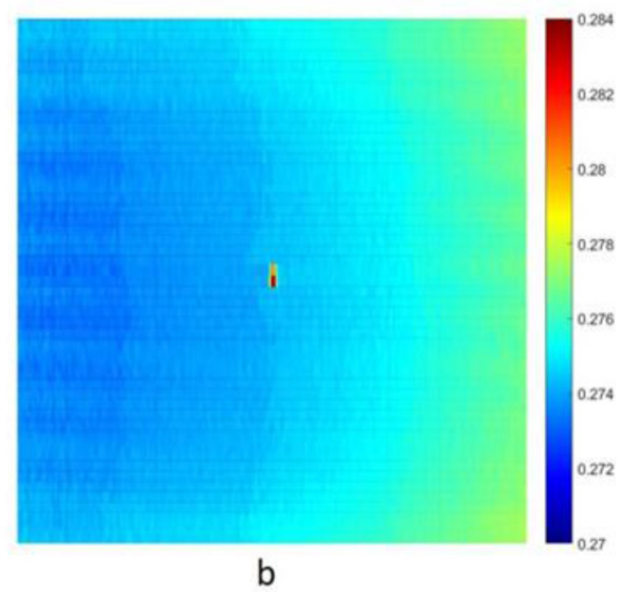

Figure 1: a) The $\mu_{H d}$ matrix in mid-plane b) scatter-to-primary ratio in mid-plane

tion contribution to the transmitted dose maps.

\section{Off-axis correction for EPID}

As the off-axis response of EPID pixels is different, a correction should be applied to obtain the correct transmitted dose. Figure 2 (a) and (b) represent the normalized EPID response and attenuation matrixes for $20 \mathrm{~cm}$ slabs, respectively. By multiplying these two matrixes, the off-axis response of EPID was obtained.
D. Back-Projection and verification Figure 3 illustrates the dose matrix extracted from TPS. The corresponding back projected dose was originated from an EPI with the same beam configuration. Gamma index with $3 \mathrm{~mm}$ distance to agreement (DTA) and 3\% dose difference was used to compare the TPS calculated dose with the EPID dose maps. In $94.89 \%$ of the points, the gamma value was less than $1((\gamma)<1)$, that indicates the acceptance criteria to be passed. 

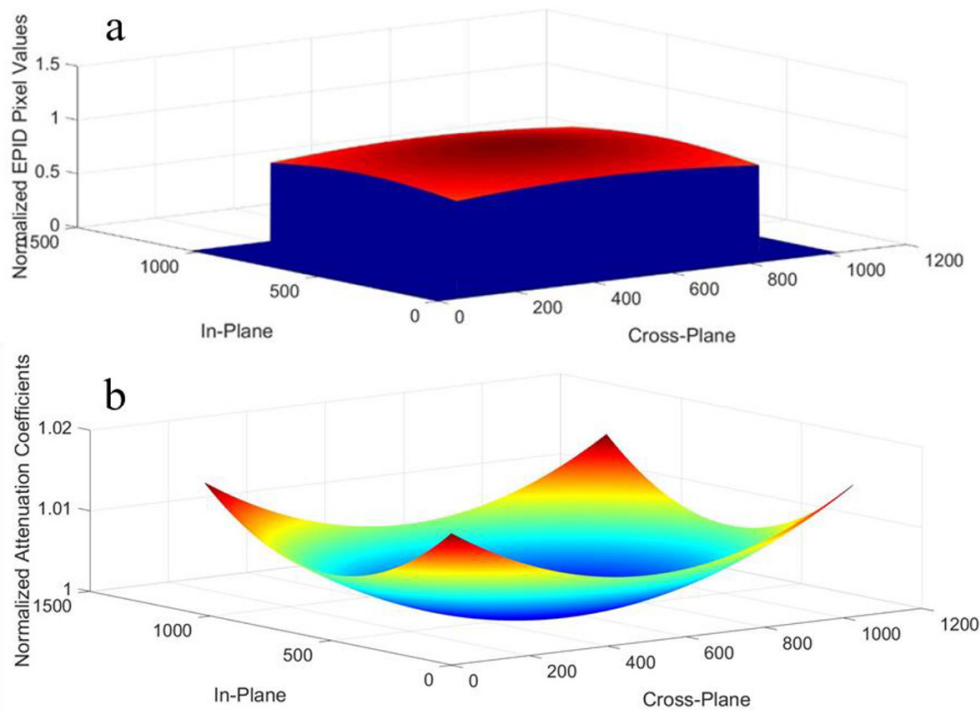

Figure 2: a) The 2D matrix of EPID response for a $25 \times 25 \mathrm{~cm}^{2}$ field size. b) The normalized attenuation matrix

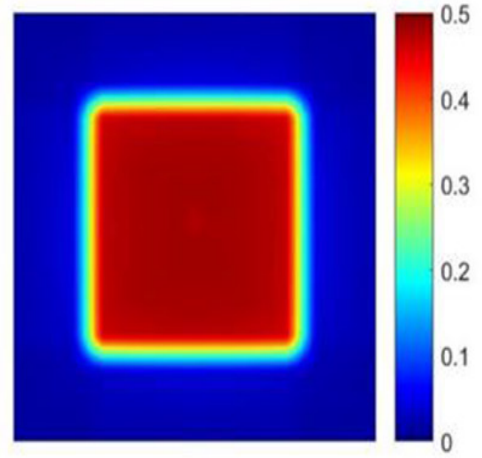

a

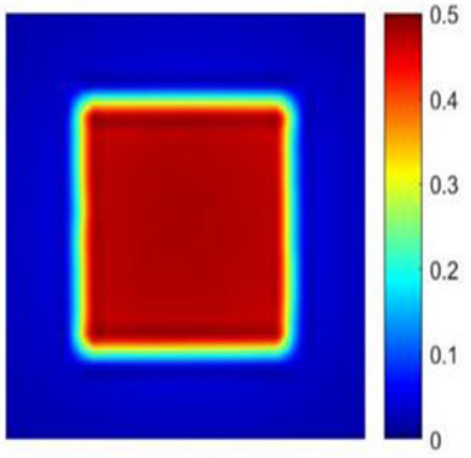

b

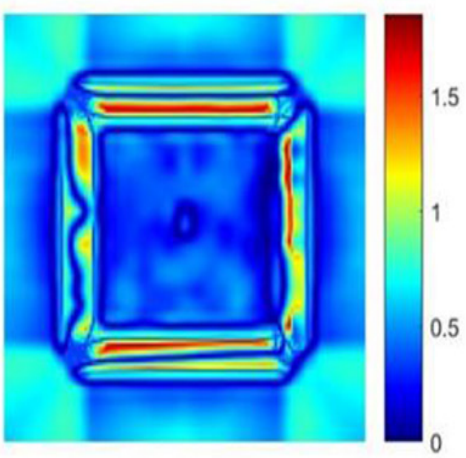

C

Figure 3: The dose matrices arising from a) TPS, b) EPID and c) Gamma comparison results.

\section{Discussion}

In the present study, the two-dimensional dose distribution at the EPID position and back-projected into the mid-plane in the homogeneous medium were obtained.

According to the results, one of the main differences between the TPS measurements and those obtained in the present study, regarding the dose distribution, was related to the shoulder and penumbra regions. No proper judgment can be made in these areas. In the same vein, Tan et al. stated that the TPS cannot ac- curately calculate the dose in shoulder and penumbra regions [13]. However, other researchers, evaluating the accuracy of dose calculation by different TPSs, have confirmed the inaccuracy of these systems [27], the various calculation algorithms have different levels of inaccuracy [28]. Therefore, the assessment of these regions was made using the GAFCHROMIC EBT3 film as an independent tool. The results of this assessment are shown in Figure 4.

Based on the results published before, the 


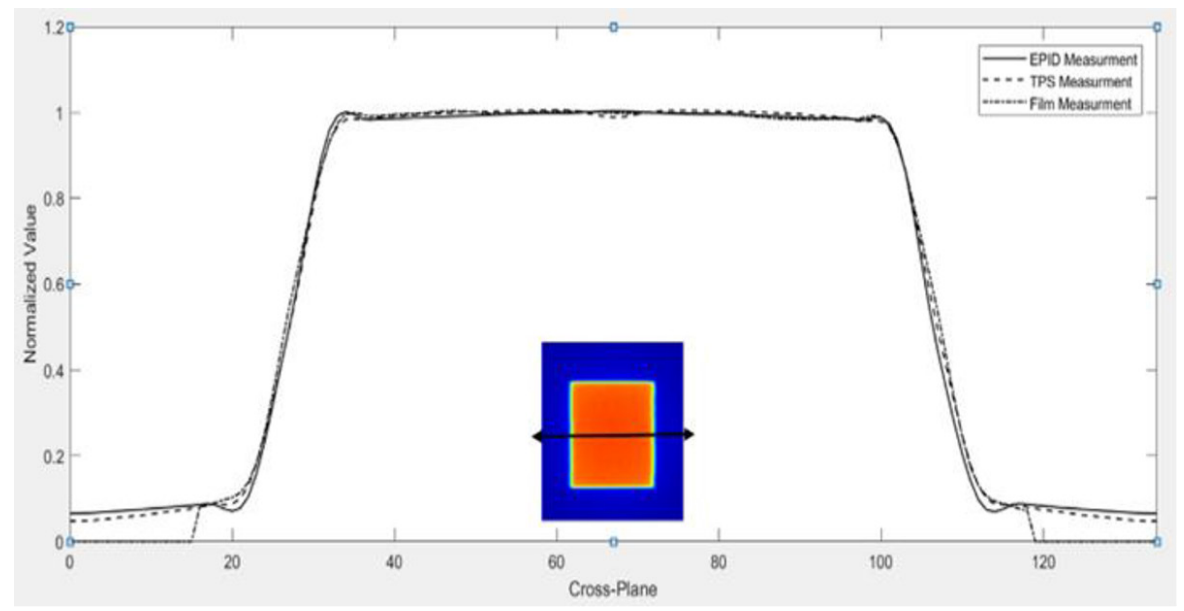

Figure 4: Dose profile (normalized to the central axis) comparisons between TPS, EPID and GAFCHROMIC EBT3 film results in the slab phantom.

behavior of the EPID calibration curve for converting the pixel values to dose is linear [29-32] and in the current study, similar results were obtained. To solve the problem of the off-axis response of the a-Si EPID, some researchers used a copper plate with different thicknesses placed on the EPID surface [9,33-35]. The clinical implementation of the method has some problems. For example, in the gantry angle of $180^{\circ}$, an air gap may be created between the copper plate and the EPID, or the copper plate may fall down due to gravity. In the current study, the copper plate was not used, and the responses of all EPID pixels were determined independently. According to the previous investigations on the reproducibility of the a-Si EPIDs response [7,10], the calculations of all EPID pixels such as SPR, $\mu_{\mathrm{Hd}}$ and the back projection, were performed independently due to the stable response of all pixels in order to achieve the dose distribution. The calibration of EPID was performed for 6 MV photon beam and $400 \mathrm{MU} / \mathrm{min}$ dose rate. The beam divergence should also be considered in all calculations.

Elekta with the collaboration of Netherlands Cancer Institute has recently introduced a solution (iViewDose, Elekta AB, Stockholm,
Sweden) that used a convolution model for transmission EPID dosimetry [36]. However, the empirical methods are more practical for clinical implementation, compared with the convolution model $[13,37,38]$. In the present study, it was attempted to verify the a-Si EPID for dosimetry purposes using an empirical method.

One of the main differences of the empirical method employed in this research with those presented in other studies is that the calculation of dose does not limit to one or two situations $[13,39]$ and that our method can calculate the $2 \mathrm{D}$ dose map at any SID.

\section{Conclusion}

In recent years, the inherent complexity of advanced treatment techniques requires new dosimetry tool for quality assurance. The presented method is not time-consuming and does not require high-speed computers, which allows the user to calculate the dose map easily at any SID and angle. However, in the first step, the method was an attempt for an angle of $0^{\circ}$ in an AP view and all measurements were made using the SAD technique. In conclusion, the method presented in this study can facilitate the determination of a 2D dose dis- 
tribution in a short period in the homogeneous phantom.

\section{Acknowledgment}

The authors would like to thank the office of Vice President for Research of Mashhad University of Medical Sciences, Mashhad, Iran for funding this work. The results described in this paper were part of student thesis.

\section{Conflict of Interest}

None

\section{References}

1. Van Elmpt W, McDermott L, Nijsten S, Wendling M, Lambin P, Mijnheer B. A literature review of electronic portal imaging for radiotherapy dosimetry. Radiother Oncol. 2008;88:289-309. doi: 10.1016/j. radonc.2008.07.008. PubMed PMID: 18706727.

2. Mohammadi M, Bezak E. Two-dimensional transmitted dose measurements using a scanning liquid ionization chamber EPID. Phys Med Biol. 2006;51:297185. doi: 10.1088/0031-9155/51/11/019. PubMed PMID: 16723778.

3. Mohammadi M, Bezak E. Evaluation of MLC leaf positioning using a scanning liquid ionization chamber EPID. Phys Med Biol. 2007;52:N21-33. doi: 10.1088/0031-9155/52/1/N03. PubMed PMID: 17183123.

4. Chen J, Chuang CF, Morin O, Aubin M, Pouliot J. Calibration of an amorphous-silicon flat panel portal imager for exit-beam dosimetry. Med Phys. 2006;33:584-94. doi: 10.1118/1.2168294. PubMed PMID: 16878562.

5. Nicolini G, Fogliata A, Vanetti E, Clivio A, Cozzi L. GLAaS: an absolute dose calibration algorithm for an amorphous silicon portal imager. Applications to IMRT verifications. Med Phys. 2006;33:2839-51. doi: 10.1118/1.2218314. PubMed PMID: 16964860.

6. Nijsten SM, Van Elmpt WJ, Jacobs M, Mijnheer BJ, Dekker AL, Lambin P, et al. A global calibration model for a-Si EPIDs used for transit dosimetry. Med Phys. 2007;34:3872-84. doi: 10.1118/1.2776244. PubMed PMID: 17985633.

7. Louwe RJ, McDermott LN, Sonke JJ, Tielenburg R, Wendling M, Van Herk M, et al. The long-term stability of amorphous silicon flat panel imaging devices for dosimetry purposes: Stability of EPID response. Med Phys. 2004;31:2989-95. doi: 10.1118/1.1803751.

8. McDermott LN, Wendling M, Van Asselen B, Stroom J, Sonke JJ, Van Herk M, et al. Clinical experience with EPID dosimetry for prostate IMRT pre-treatment dose verification. Med Phys. 2006;33:3921-30. doi: 10.1118/1.2230810. PubMed PMID: 17089854.

9. McDermott LN, Louwe RJ, Sonke JJ, Van Herk MB, Mijnheer BJ. Dose-response and ghosting effects of an amorphous silicon electronic portal imaging device. Med Phys. 2004;31:285-95. doi: 10.1118/1.1637969. PubMed PMID: 15000614.

10. Winkler P, Hefner A, Georg D. Dose-response characteristics of an amorphous silicon EPID. Med Phys. 2005;32:3095-105. doi: 10.1118/1.2040711. PubMed PMID: 16279061.

11. Mijnheer B, Beddar S, Izewska J, Reft C. In vivo dosimetry in external beam radiotherapy. Med Phys. 2013;40:070903. doi: 10.1118/1.4811216. PubMed PMID: 23822404.

12. Essers M, Mijnheer BJ. In vivo dosimetry during external photon beam radiotherapy. Int J Radiat Oncol Biol Phys. 1999;43:245-59. doi: 10.1016/s03603016(98)00341-1.PubMed PMID: 10030247.

13. Tan YI, Metwaly M, Glegg M, Baggarley SP, Elliott A. A dual two dimensional electronic portal imaging device transit dosimetry model based on an empirical quadratic formalism. Br J Radiol. 2015;88:20140645. doi: 10.1259/bjr.20140645. PubMed PMID: 25969867; PubMed Central PMCID: PMC4628518.

14. Pecharroman-Gallego R, Mans A, Sonke JJ, Stroom JC, Olaciregui-Ruiz I, Van Herk M, et al. Simplifying EPID dosimetry for IMRT treatment verification. Med Physs. 2011;38:983-92. doi: 10.1118/1.3547714. PubMed PMID: 21452735.

15. Heijmen BJ, Pasma KL, Kroonwijk M, Althof VG, de Boer JC, Visser AG, et al. Portal dose measurement in radiotherapy using an electronic portal imaging device (EPID). Phys Med Biol. 1995;40:1943-55. doi: 10.1088/0031-9155. PubMed PMID: 8587942.

16. McCurdy BM, Pistorius S. A two-step algorithm for predicting portal dose images in arbitrary detectors. Med Phys. 2000;27:2109-16. doi: 10.1118/1.1289375. PubMed PMID: 11011740.

17. Pasma KL, Kroonwijk M, De Boer JC, Visser AG, Heijmen BJ. Accurate portal dose measurement with a fluoroscopic electronic portal imaging device (EPID) for open and wedged beams and dynamic multileaf collimation. Phys Med Biol. 1998;43:2047-60. doi: 10.1088/0031-9155/43/8/004. PubMed PMID: 9725588.

18. Pasma KL, Kroonwijk M, De Boer JC, Visser AG, Heijmen BJ. Accurate portal dose measurement with a fluoroscopic electronic portal imaging device (EPID) for open and wedged beams and dynamic multileaf collimation. Phys Med Biol. 1998;43:2047-60. PubMed PMID: 9725588.

19. Boellaard R, Essers M, Van Herk M, Mijnheer BJ. New method to obtain the midplane dose us- 
ing portal in vivo dosimetry. Int $J$ Radiat Oncol Biol Phys. 1998;41:465-74. doi: 10.1016/s03603016(98)00048-0 .PubMed PMID: 9607366.

20. Wendling $M$, McDermott $L N$, Mans A, Sonke JJ, Van Herk M, Mijnheer BJ. A simple backprojection algorithm for 3D in vivo EPID dosimetry of IMRT treatments. Med Phys. 2009;36:3310-21. doi: 10.1118/1.3148482. PubMed PMID: 19673227.

21. Baek TS, Chung EJ, Son J, Yoon M. Feasibility study on the verification of actual beam delivery in a treatment room using EPID transit dosimetry. Radiat Oncol. 2014;9:273. doi: 10.1186/s13014-014-0273-8. PubMed PMID: 25472838; PubMed Central PMCID: PMC4262986.

22. IviewGT E. Corrective maintenance manual. Elekta Limited. 2006.

23. Cilla $S$, Azario L, Greco F, Fidanzio A, Porcelli $A$, Grusio $\mathrm{M}$, et al. An in-vivo dosimetry procedure for Elekta step and shoot IMRT. Phys Med. 2014;30:41926. doi: 10.1016/j.ejmp.2013.11.005. PubMed PMID: 24361278.

24. Deshpande S, Xing A, Holloway L, Metcalfe P, Vial P. Dose calibration of EPIDs for segmented IMRT dosimetry. J Appl Clin Med Phys. 2014;15:4895. doi: 10.1120/jacmp.v15i6.4895. PubMed PMID: 25493513; PubMed Central PMCID: PMC5711103.

25. Bjarngard BE, Shackford H. Attenuation in high-energy x-ray beams. Med Phys. 1994;21:1069-73. doi: 10.1118/1.597349. PubMed PMID: 7968838.

26. Wang KK, Zhu TC. Modeling scatter-to-primary dose ratio for megavoltage photon beams. Med Phys. 2010;37:5270-8. doi: 10.1118/1.3480480. PubMed PMID: 21089761.

27. Tan YI, Metwaly M, Glegg M, Baggarley S, Elliott A. Evaluation of six TPS algorithms in computing entrance and exit doses. J Appl Clin Med Phys. 2014;15:229-40. doi: 10.1120/jacmp.v15i3.4739. PubMed PMID: 24892349; PubMed Central PMCID: PMC5711058.

28. Golestani A, Houshyari M, Mostaar A, Arfaie AJ. Evaluation of dose calculation algorithms of Isogray treatment planning system using measurement in heterogeneous phantom. Reports of Radiotherapy and Oncology. 2015;2(3): e5320. doi: 10.17795/rro-5320.

29. Mhatre V, Pilakkal S, Chadha P, Talpatra K. Dosimetric Comparison of a-Si 1200 and a-Si 1000 Electronic Portal Imager for Intensity Modulated Radiation Therapy (IMRT). J Nucl Med Radiat Ther. 2018;9:2. doi: 10.4172/2155-9619.1000354.

30. Camilleri J, Mazurier J, Franck D, Dudouet P, Latorzeff I, Franceries X. 2D EPID dose calibration for pretreatment quality control of conformal and IMRT fields: A simple and fast convolution approach. Phys Med.
2016;32:133-40. doi: 10.1016/j.ejmp.2015.10.094. PubMed PMID: 26573130.

31. Grządziel A, Smolińska B, Rutkowski R, Ślosarek K. EPID dosimetry-configuration and pre-treatment IMRT verification. Reports of Practical Oncology \& Radiotherapy. 2007;12:307-12. doi: 10.1016/s15071367(10)60069-7.

32. Renner WD, Norton K, Holmes T. A method for deconvolution of integrated electronic portal images to obtain incident fluence for dose reconstruction. $J$ Appl Clin Med Phys. 2005;6:22-39. doi: 10.1120/jacmp.2026.25359. PubMed PMID: 16421498; PubMed Central PMCID: PMC5723452.

33. Greer PB. Off-axis dose response characteristics of an amorphous silicon electronic portal imaging device. Med Phys. 2007;34:3815-24. doi: 10.1118/1.2779944. PubMed PMID: 17985627.

34. Olaciregui-Ruiz I, Rozendaal R, Mijnheer B, Van Herk $\mathrm{M}$, Mans A. Automatic in vivo portal dosimetry of all treatments. Phys Med Biol. 2013;58:8253-64. doi: 10.1088/0031-9155/58/22/8253. PubMed PMID: 24201085.

35. Parent L, Fielding AL, Dance DR, Seco J, Evans PM. Amorphous silicon EPID calibration for dosimetric applications: comparison of a method based on Monte Carlo prediction of response with existing techniques. Phys Med Biol. 2007;52:3351-68. doi: 10.1088/00319155/52/12/003. PubMed PMID: 17664548.

36. Kydonieos $M$, Folgueras $A$, Florescu L, Cybulski $T$, Marinos N, Thompson G, et al. TH-AB-201-10: Portal Dosimetry with Elekta IViewDose: Performance of the Simplified Commissioning Approach Versus Full Commissioning. Med Phys. 2016;43:3851. doi: 10.1118/1.4958039.

37. Mohammadi M, Bezak E. Evaluation of relative transmitted dose for a step and shoot head and neck intensity modulated radiation therapy using a scanning liquid ionization chamber electronic portal imaging device. J Med Phys. 2012;37:14-26. doi: 10.4103/09716203.92716. PubMed PMID: 22363108; PubMed Central PMCID: PMC3283912.

38. Mohammadi M, Bezak E, Reich P. Verification of dose delivery for a prostate sIMRT treatment using a SLIC-EPID. App/ Radiat Isot. 2008;66:1930-8. doi: 10.1016/j.apradiso.2008.04.024. PubMed PMID: 18583141.

39. Kavuma A, Glegg M, Metwaly M, Currie G, Elliott A. A novel method for patient exit and entrance dose prediction based on water equivalent path length measured with an amorphous silicon electronic portal imaging device. Phys Med Biol. 2010;55:435-52. doi: 10.1088/0031-9155/55/2/007. PubMed PMID: 20019398. 